University of Nebraska - Lincoln

DigitalCommons@University of Nebraska - Lincoln

Educational Psychology Papers and

Publications

Educational Psychology, Department of

January 2006

\title{
Promoting Parent Use of Empirically Supported Reading Interventions: Two Experimental Investigations of Child Outcomes
}

\author{
Michael Persampieri \\ University of Nebraska-Lincoln \\ Valerie Gortmaker \\ University of Nebraska-Lincoln \\ Edward J. Daly III \\ University of Nebraska-Lincoln, edaly2@unl.edu \\ Susan M. Sheridan \\ University of Nebraska-Lincoln, ssheridan2@unl.edu \\ Merilee McCurdy \\ University of Nebraska-Lincoln, mmccurdy2@unl.edu
}

Follow this and additional works at: https://digitalcommons.unl.edu/edpsychpapers

Part of the Educational Psychology Commons

Persampieri, Michael; Gortmaker, Valerie; Daly, Edward J. III; Sheridan, Susan M.; and McCurdy, Merilee, "Promoting Parent Use of Empirically Supported Reading Interventions: Two Experimental Investigations of Child Outcomes" (2006). Educational Psychology Papers and Publications. 2.

https://digitalcommons.unl.edu/edpsychpapers/2

This Article is brought to you for free and open access by the Educational Psychology, Department of at DigitalCommons@University of Nebraska - Lincoln. It has been accepted for inclusion in Educational Psychology Papers and Publications by an authorized administrator of DigitalCommons@University of Nebraska - Lincoln. 


\title{
Promoting Parent Use of Empirically Supported Reading Interventions: Two Experimental Investigations of Child Outcomes
}

\author{
Michael Persampieri, Valerie Gortmaker, Edward J. Daly III*, \\ Susan M. Sheridan and Merilee McCurdy \\ University of Nebraska - Lincoln, USA \\ *Corresponding author. E-mail: edaly2@unl.edu
}

Two experimental investigations of the effects of parent delivered reading interventions were conducted. Tutoring packages consisting of empirically supported intervention components were delivered by parents for at least several weeks after initial parent training. Both experiments used single-case experimental designs and measured participants' oral reading fluency in passages. Experiment 1 used a multiple-probe design across tasks (passages) to evaluate tutoring effects for two students with learning disabilities. Results indicate that both students increased their reading fluency and maintained those increases over time. Experiment 2 used a brief experimental analysis that included both experimenter and parent delivered instructional trials to validate the treatment package. Next, the treatment package was evaluated using an alternating treatments design. Results were uniformly positive. An interesting but not surprising correlation was also found between treatment integrity and student outcomes. Results are discussed in terms of the framework and skills that behavior analysts have for working with parents and schools to improve their children's academic responding.

\section{INTRODUCTION}

National assessments of students' reading skills reveal that the number of students who have poor reading skills is overwhelming. Based on a recent, large national sample, less than one-third of fourth grade students read proficiently at grade level (National Center for Education Statistics; NCES, 2003). If they fail to read by fourth grade, children have a "future of diminished success" (US Department of Education, 2001). Poor reading skills will not only have a detrimental effect on students' academic trajectory; they also have been associated with behavioral and emotional problems such as aggressive behavior 
and poor self-concept (Good, Simmons, \& Smith, 1998), as well as high dropout rates (Juel, 1988). According to one source, over 40 million adults in America are illiterate and the economic impact represents millions of lost dollars due to low productivity, accidents, and errors (Adult Literacy Service, 2004). It appears, therefore, that America's literacy landscape will not be getting better any time soon without broad and effective intervention of some type.

Although the ultimate goal of reading instruction is comprehension, students with reading problems often have difficulties with reading fluency, a prerequisite to independent comprehension (Chard, Vaughn, \& Tyler, 2002). Reading fluency has been characterized by National Institute of Child Health and Human Development (2000) as "a neglected reading skill in America's classrooms" (p. 3.1). Since poor readers and those identified with a learning disability start out with slower reading rates, their skills increase at a slower rate (Good et al., 1998). Deficiencies in oral reading fluency will therefore adversely affect comprehension and consequently slow down mastery of content areas like science and social studies.

The benefits of parent involvement in reading with their child are undisputed (Epstein, 1996). Home support for reading has been researched extensively (cf., Payne, Whitehurst, \& Angell, 1994; Storck \& Whitehurst, 2001), and has yielded highly consistent findings. Regardless of economic, racial, ethnic and educational backgrounds, educational benefits are enhanced when families engage in reading activities with their child (Epstein, 1996). Conversely, poor readers have few early reading-related experiences. Despite these benefits, the effectiveness of parents' involvement in actively teaching their child reading skills has not been researched extensively.

Parents are major stakeholders in children's education (Christenson \& Buerkle, 1999) and have great potential for contributing to the academic success of their children (Hook \& DuPaul, 1999). Parent-directed interventions provide opportunities to extend the learning environment (Christenson \& Sheridan, 2001), since parents can offer one-on-one attention and make immediate modifications when needed (Leach \& Siddal, 1990). With adequate support, parents have been shown to be effective participants in the academic intervention process (Duvall \& Ward, 1997; Galloway \& Sheridan, 1994; Weiner, Sheridan, \& Jenson, 1998). However, parents often do not know how to help their child academically and may feel inadequate in doing so (Wolfendale, Topping, \& Hewison, 1986). Indeed, without specific, structured interventions, parents often feel ill-equipped to help their child learn to read.

Parent-tutoring with reading strategies has yielded increases in reading fluency (Fiala \& Sheridan, 2003; Hook \& DuPaul, 1999; Wilks \& Clarke, 1988) and comprehension (Wilks \& Clarke, 1988). Parents have effectively used a variety of methods, including modeling, performance feedback, error correction, 
repeated readings, and direct instruction. For instance, Hook and DuPaul (1999) trained parents to use repeated readings, error correction, and a reward system to tutor children with ADHD. Reading fluency increased across home and school settings. Parents and students both reported that the lessons were "enjoyable" and manageable. In parent tutoring, procedural checklists, audiotaping, videotaping, and phone calls have been used to support parents in the correct implementation of procedures (Hook \& DuPaul, 1999; Powell-Smith, Stoner, Shinn, \& Good, 2000; Wilks \& Clarke, 1988). Monitoring procedures alone, however, is probably not sufficient to achieve high treatment integrity. Parents need to learn how to use the procedures correctly. To this end, direct training methods like modeling and feedback are good candidates for teaching parents to do interventions correctly (Sterling-Turner, Watson, Wildmon, Watkins, \& Little, 2000). With adequate training and support parents' confidence increases and they are more likely to implement the prescribed treatment correctly (Wolfendale, Topping, \& Hewison, 1986).

The use of evidence based interventions has emerged as the new standard for increasing children's achievement (Kratochwill \& Stoiber, 2002; U.S. Department of Education's Institute of Education Sciences, 2004). In the area of reading, The National Reading Panel (2000) found that guided oral reading interventions have a consistent, positive impact on word recognition, fluency, and comprehension. Instructional components that can serve to structure guided practice include interventions such as listening passage preview (reading to the student), repeated readings (having the student repeatedly practice a story), error correction, performance feedback, and reward contingencies. All have proven effective at increasing reading rates (Chard et al., 2002; Daly, Lentz, \& Boyer, 1996; Daly \& Martens, 1994; Sindelar, Monda, \& O'Shea, 2001).

An intervention's effectiveness is not solely dependent on proper implementation of an evidence based intervention, however. An intervention that has been shown to be effective in the research may not be appropriate for every student. In other words, empirically supported interventions themselves do not guarantee success. The intervention must be validated locally through a direct demonstration of behavior change on the part of the student in question. A brief experimental analysis (Daly, Martens, Hamler, Dool, \& Eckert, 1999) may help a practitioner to validate a potential treatment before recommending that parents apply the treatment for an extended period of time. Using single-case experimental designs with abridged data series, repeated measures, and empirically supported interventions as brief instructional trials, a number of investigations have identified interventions that lead to rapid increases in oral reading fluency on a case-by-case basis (i.e., Daly et al., 1999; Daly, Shroder, \& Robinson, 2001; Daly, Murdoch, Lillenstein, Webber, \& Lentz, 2002; Eckert, Ardoin, Daly, \& Martens, 2002; Noell, Freeland, Witt, \& Gansle, 2001; Noell et al., 1998). 
Recently, Valleley, Evans, and Allen (2002) used brief tests of reading interventions (instructional trials) to identify a strategy that was subsequently used by the parent of a child with learning disabilities. As in the other studies, the therapist conducted the instructional trials. A brief experimental analysis, however, could readily be extended to observations of the parent directly implementing an effective treatment as a part of supportive training. There are several advantages to this approach. First, after an effective treatment is identified, the therapist can directly observe the parent using the intervention. Positive and corrective feedback can be given to the parent following training. Also, parents directly experience a previously validated treatment, putting them into contact with contingencies that are presumably reinforcing (seeing their child read better in a tutoring session). The brief nature of the validation process makes it efficient and the use of a controlled experimental design increases confidence in the effectiveness of the intervention.

The purpose of the two studies reported here was to increase student reading fluency by training parents to implement empirically supported interventions. Experiment 1 examined the effects of parent tutoring with two children with learning disabilities utilizing a multi-component treatment package that was taught to the parents. Using a multiple-probe design across tasks (reading passages), the study examined changes in reading fluency across time within passages as a result of parent tutoring. Experiment 2 also examined the effects of parent tutoring interventions. Prior to recommending and training the parent in the use of an intervention package, however, the intervention was validated through a brief experimental analysis. The brief experimental analysis also included parent administered conditions to determine whether parents could obtain the same increase in responding that had been achieved when the experimenter conducted sessions. Using an alternating treatments design, the parent tutoring package was evaluated relative to a control condition.

\section{EXPERIMENT 1}

\section{Method}

\section{Participants and Setting}

Two students enrolled in a Midwestern public school participated in this study. Both students had previously been identified with a learning disability in reading and were referred by their teachers for reading interventions. Martin was a 9year and 8month old, bi-racial (African American/Caucasian) male who had just completed third grade and Tom was an 8-year and 4-month old, Arab-American male who had just completed second grade. Tutoring sessions occurred during 
the summer months at each child's home and sessions were conducted by the child's parent. Additionally, both children were individually assessed three times per week at the school. Throughout the duration of the study, participants were not receiving reading assistance from paid tutors, nor did they engage in summer reading programs. (The students were brought to the school by their parents for assessment purposes only.)

\section{Materials}

Parents were provided with a audiocassette recorder and tape, copies of the tutoring passages (described below), and a tutoring protocol (available from the third author upon request).

Assessment Passages. Assessment passages were the three passages for each student in which outcomes were measured and in which a reward contingency was offered for improving accurate and fluent reading during assessments. These passages were based on the tutoring passages taken from the Silver, Burdett, and Ginn (Pearson et al., 1989) reading series (described next). Each tutoring passage was rewritten to create an assessment passage that was a new story with many of the same words. Six randomly chosen passages were selected initially. Passages were approximately 100 words in length. The three most appropriate passages (based on screening results, described below) of the six original passages were selected for each student. Readability scores for the assessment passages were computed using the Spache formula (1953). The average readability for the first grade passages was 1.6 (range, 1.4 to 1.8 ) for Tom and 2.7 (range, 2.3 to 3.0) for Martin.

Tutoring Passages. Tutoring passages were stories taken from the Silver, Burdett, and Ginn basal reading series (Pearson et al., 1989). Only narrative and expository texts were used. Passages contained approximately 100 words. The average readability of the passages was 1.8 (range, 1.5 to 1.9) for Tom and 2.6 (range, 2.3 to 2.9) for Martin. Each passage had a high level of word overlap (Daly, Martens, Kilmer, \& Massie, 1996) with one of the assessment passages, but was written as a separate story. To determine the percentage of word overlap, the number of words appearing in both passages was divided by the total number of words in the assessment passage in order to calculate the percentage of word overlap. Average overlap was $87.9 \%$ for Tom (range, $85.7 \%$ to $90.5 \%$ ) and $84.6 \%$ for Martin (range, $80.5 \%$ to $87.2 \%$ ). Tutoring passages differed from assessment passages to avoid direct overlap between training and assessment conditions. In this way, assessments would indicate the degree of stimulus control achieved under similar but not identical training conditions, an index of generalization of behavior. 


\section{Dependent Variables and Interobserver Agreement}

Correctly read words per min (CRW/min) and errors per minute were measured to examine the effects of tutoring on reading performance in the assessment passages. A correctly read word was defined as a word that is pronounced correctly in 3 s (Shinn, 1989). A word was scored as an error if the student omitted, mispronounced, substituted, or paused for more than $3 \mathrm{~s}$. The number of CRW and errors per min were calculated for each assessment passage.

An audiocassette recorder was used during all sessions to assess interobserver agreement. An independent observer listened to $83 \%$ (i.e., 25 sessions) of the audiotaped reading assessments (randomly chosen) conducted by the experimenter. The reviewer independently scored each assessment for CRW and errors. Interobserver agreement was calculated by dividing the number of agreements by the number of agreements plus disagreements and multiplying the result by 100 to obtain a percentage. The mean interobserver agreement was $97 \%$ (range, $91 \%$ to $100 \%$ ).

Parent ratings on the Behavior Intervention Rating System (BIRS; Von Brock \& Elliott, 1987) were obtained to assess the parents' perceptions of the clinical importance of the participants' reading trajectory. The BIRS is a 24-item scale that assesses perceptions for the factors of Acceptability, Effectiveness, and Time to Effect.

\section{Pre-experimental Screening and Selection of Stimulus Materials}

Both participants were initially screened to determine an appropriate instructional level for tutoring activities. Using an independent set of randomly selected passages from the Silver, Burdett, and Ginn reading series, the experimenter had each child read three grade level passages from each grade level, first through third. The grade level at which students were reading within a range of 40 to $60 \mathrm{CRW} / \mathrm{min}$ (Deno \& Mirkin, 1977) was chosen as the level at which students would be tutored. After the instructional level was determined (first grade for Tom and third grade for Martin), repeated assessments were performed on six randomly chosen passages with reading passages from that level. The three passages which were closest in CRW and errors per min and which had the most stable baselines for each participant (based on 3 data points for each passage) were chosen for use in the study. The three passages were randomly assigned to one of the three treatment weeks.

\section{Experimental Design and Procedures}

A multiple-probe design across tasks (reading passages) was used to evaluate the results of the reading program. Using this design, experimental control was established when a change in level, trend, and/or variability occurs only under 
the condition (reading passage) receiving treatment (parent tutoring). All other baseline data patterns should remain stable. Repeated measurements are carried out across passages initially to obtain baselines on all passages. Then, repeated measurements across passages continue while treatment is introduced sequentially across passages, but never concurrently. When one passage in under treatment, the other passages are under either baseline or maintenance. Once treatment is terminated for a given passage, subsequent assessments reveal maintenance of treatment effects for that passage.

Parent Training. Individual parent-tutoring training was conducted by the second author. Training was conducted in one session at the child's school. The training session was conducted in three parts: (1) the experimenter described the steps for the reading intervention, (2) the experimenter modeled the intervention for the parent with the child, and (3) the experimenter observed the parent practice the tutoring procedure and provided feedback. Parents were required to perform the intervention with $100 \%$ accuracy in the presence of the experimenter before the training was terminated. A structured protocol was provided to the parents. Parents were instructed to engage in the tutoring procedures 3 to 5 days a week for 10 to $15 \mathrm{~min}$ per session.

Reading Intervention. The reading intervention included repeated readings (RR; Rashotte \& Torgesen, 1985), error correction with sentence repeat (O'Shea, Munson, \& O'Shea, 1984), and a contingent reward for improvements in performance (Noell et al., 1998). At the beginning of each session, the participant was asked to read one tutoring passage aloud to his parents. The parent noted errors while the child was reading. Following the initial reading, parents modeled the accurate pronunciation of any mispronounced words. Then the child was asked to repeat the word and read the entire sentence again once. Parents were instructed to praise their child when he correctly read a sentence that had previously contained one or more errors. Participants then re-read the same passage until the 10 to 15 minute parent-tutoring session ended. After the parent-tutoring session, participants placed a sticker on their sticker calendar to denote completion of a tutoring session. Stickers on the calendar could be used toward tangible rewards in assessments with the experimenter (described next).

Assessment and Reward Condition. The experimenter met with each participant three times a week to conduct 1 min assessments on all three passages. All three passages were administered in a random order each time. During treatment weeks, tangible items (e.g., pens, balls, small games, candy, etc.) were offered for achieving performance goals during assessments. Goals were individualized an and determined based on the participant's best performance on a given passage 
to date. To earn a reward, the participant was told that he was required to read at least one $\mathrm{CRW} / \mathrm{min}$ more than his previous best performance and emit three or fewer errors. Participants were reminded about their goal prior to reading the tutoring assessment passage. Tom and Martin met their goal and received a reward $78 \%$ and $67 \%$ of the time, respectively. Additionally, each family was provided stickers and a calendar of the intervention dates. The children were rewarded each day they practiced their readings for 10 to $15 \mathrm{~min}$ by putting a sticker on their chart.

\section{Results and Discussion of Experiment 1}

\section{Treatment Integrity}

To measure treatment integrity, parents were asked to record all sessions on audiotapes. The second author listened to $40 \%$ of the parent-tutoring sessions. The percentage of intervention steps correctly implemented by the parents during the tutoring sessions was calculated based on the number of steps correctly completed by the parent divided by the total number of intervention steps that appeared on the intervention protocol. The mean number of correctly implemented steps was $92 \%$ (range, $72 \%$ to $100 \%$ ). Additionally, the sticker calendar not only served as a reward for each child, but also provided an additional integrity check. According to the students' charts, Martin's parent tutoring sessions were conducted an average of 4.7 times per week and Tom's sessions occurred an average of 5.3 times per week.

\section{Child Outcomes}

Martin's data are displayed in Figure 1 and Table 1. A dramatic change in level is visible for both CRW/min and errors in passage 1 following the introduction of treatment. The changes in level were more moderate for the remaining two passages. There was an increasing trend in passage 2 during baseline that mitigates somewhat the treatment effects observed for this passage. In other words, it is not altogether clear whether the increasing trend during treatment for passage 2 is a result of tutoring or a continuation of an already increasing trend in the data. A more modest increase during baseline is visible for passage 3 . Under treatment for this passage, however, a clear change in trend is evident. Although the increasing trends in two of the passages provoke some concern and reservation about demonstration of experimental control, it is noteworthy that all but one of the treatment data points exceed all the baseline data across passages (see the first treatment data point of passage 3 for the exception). Maintenance data suggest that treatment effects were maintained across all three passages once intervention was removed (including the reward contingency). Errors decreased for all three 


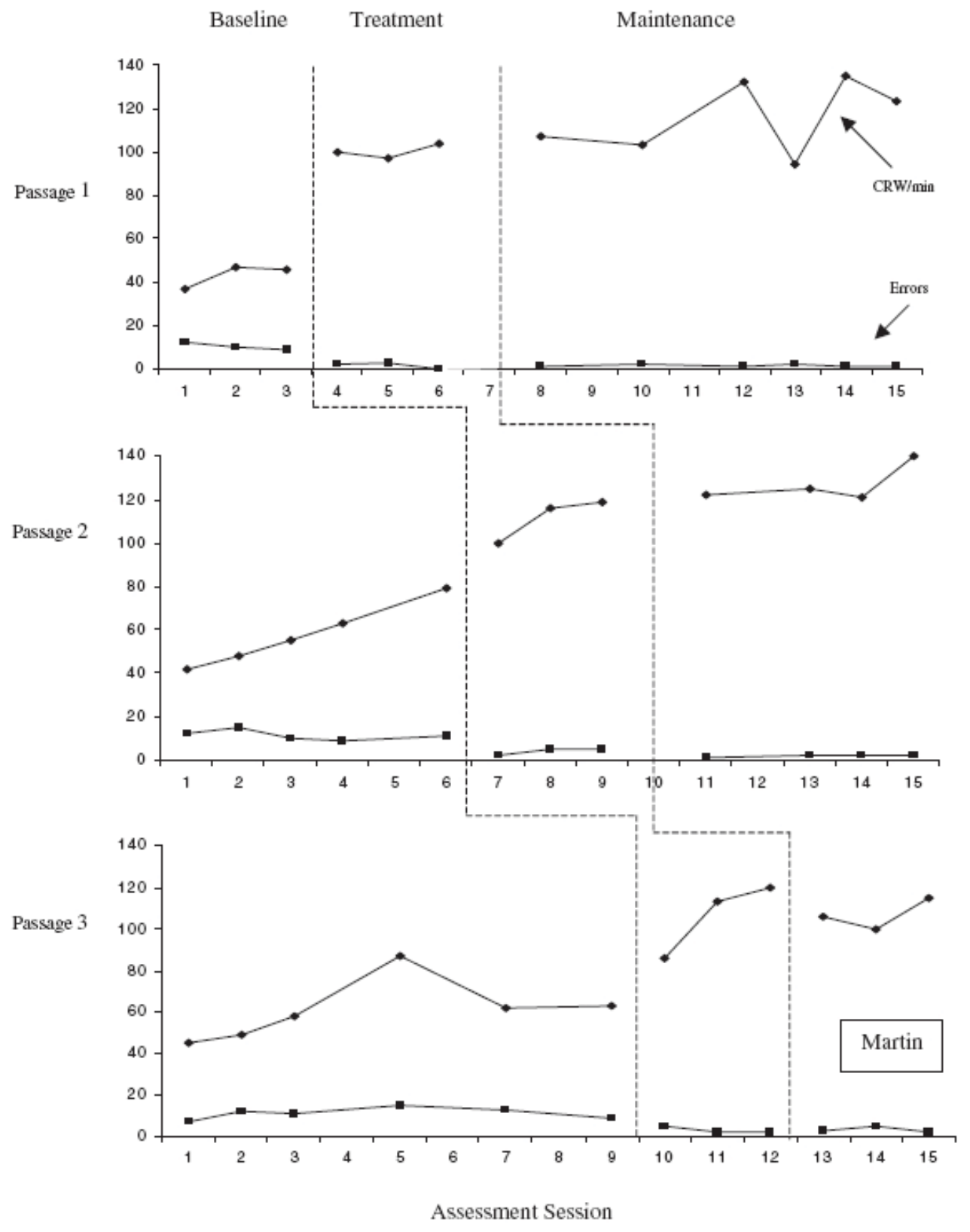

Figure 1. The number of CRW and errors per min for Martin across baseline, treatment, and followup phases. 
Table 1. Means and standard deviations for CRW and errors per min for each experimental phase in Experiment I.

\begin{tabular}{|c|c|c|c|c|c|c|c|c|c|}
\hline \multirow{3}{*}{$\begin{array}{l}\text { Participant } \\
\text { Passage }\end{array}$} & \multicolumn{9}{|c|}{ Experimental phase } \\
\hline & \multicolumn{3}{|c|}{ Baseline } & \multicolumn{3}{|c|}{ Treatment } & \multicolumn{3}{|c|}{ Maintenance } \\
\hline & $C R W$ & $S D$ & E & $C R W$ & $S D$ & $E$ & $C R W$ & $S D$ & $E$ \\
\hline \multicolumn{10}{|l|}{ Martin } \\
\hline 1 & 43.3 & 5.5 & 10.3 & 100.3 & 3.5 & 1.7 & 115.7 & 15.9 & 1.3 \\
\hline 2 & 57.4 & 9.1 & 11.4 & 111.7 & 10.2 & 4.0 & 124.0 & 8.8 & 1.8 \\
\hline 3 & 60.7 & 18.0 & 11.2 & 106.3 & 18.0 & 3.0 & 107.0 & 7.5 & 3.3 \\
\hline \multicolumn{10}{|l|}{ Tom } \\
\hline 1 & 35.7 & 11.4 & 8.7 & 78.3 & 12.7 & 2.3 & 80.0 & 10.6 & 1.8 \\
\hline 2 & 41.0 & 10.9 & 7.4 & 64.3 & 16.7 & 1.7 & 78.8 & 17.6 & 3.5 \\
\hline 3 & 59.5 & 12.4 & 6.3 & 86.3 & 2.9 & 2.2 & 94.3 & 13.6 & 5.7 \\
\hline
\end{tabular}

Note: $C R W=$ Correctly read words; $S D=$ Standard deviation; $E=$ Errors.

passages almost immediately with the introduction of treatment. Visual analysis results are corroborated by the summary statistics for CRW/min (Table 1).

A similar pattern was observed for Tom (Figure 2 and Table 1). There was a visible increase in level and trend for passage 1 following a stable baseline. Increasing trends are evident for the following two passages. Fortunately, all but one of the data points exceeds baseline for these two passages (with the second treatment data point of passage 2 being the exception). As with Martin, errors decreased immediately when treatment began for each passage and maintenance of effects is visible once treatment was withdrawn for a passage (with an exception for passages 2 and 3 during session 14). Once again, the summary statistics corroborate visual analysis results (Table 1).

Following completion of the study, each child's mother completed the BIRS. The scale items are completed using a 6-point Likert scale with a rating of "6" representing Very Acceptable or Very Effective. In general, Tom's and Martin's parents both reported that the procedures were moderately to very acceptable. Acceptability mean item scores were 3.80 and 5.93. Mean item scores suggested that parents perceived the treatment procedures to be moderately to very effective. Effectiveness mean item scores were 3.44 and 5.89. Anecdotally, in a written explanation of her scores on the BIRS, Tom's mother stated that she believed the intervention was effective, but "did not cure [her child's] learning disability."

The results of Experiment 1 were encouraging, especially in light of the high degree of treatment integrity achieved by the parents. The results generally supported the use of an intervention package that contained many empirically supported intervention components and emphasized repeated practice (opportunities to respond), error correction, and manipulation of consequences (performance feedback and reward contingencies). A unique feature of the 


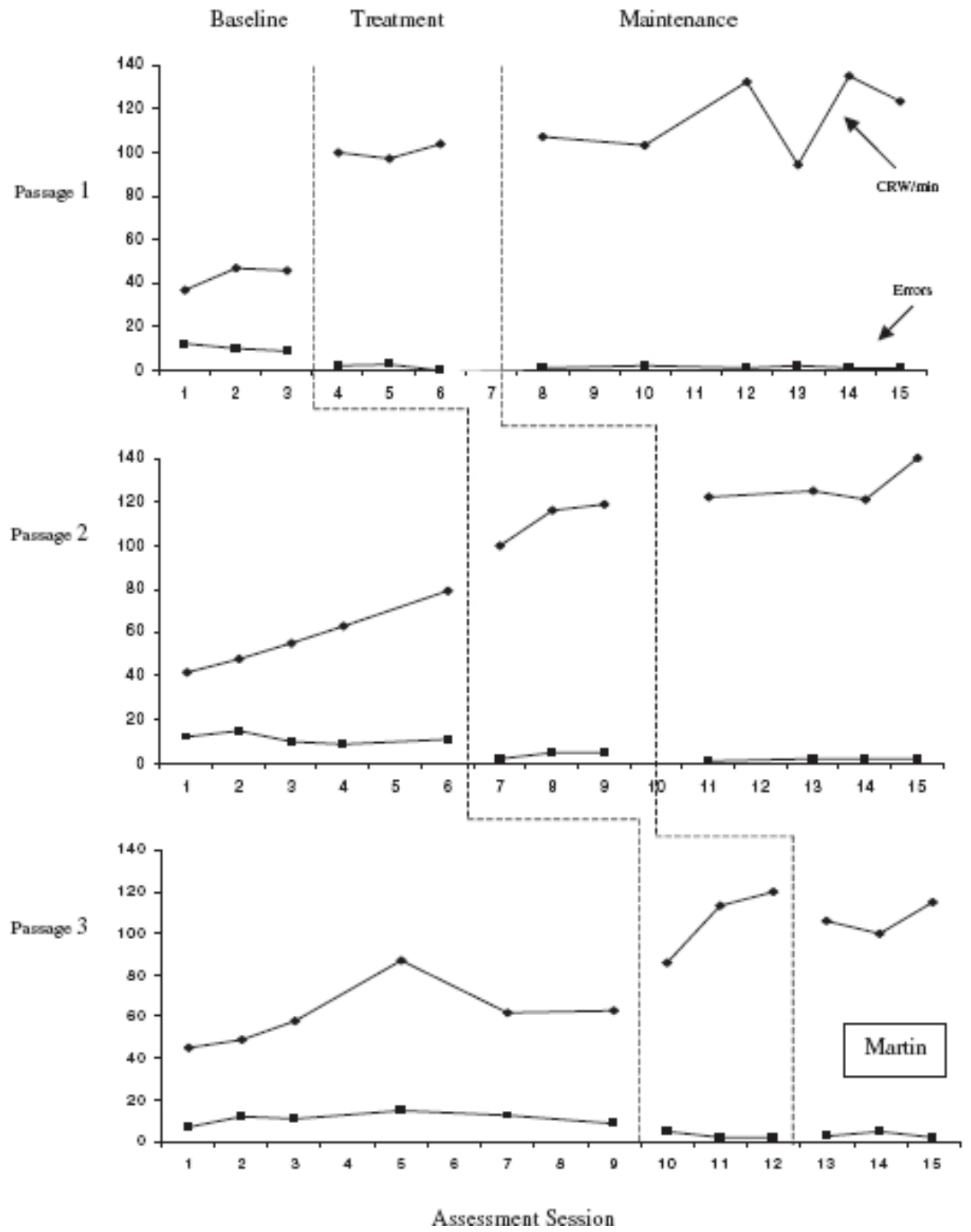

Figure 2. The number of CRW and errors per min for Tom across baseline, treatment and follow-up phases. 
multiple-probe design is that it allows for an evaluation of experimental effect over time within passages, as well as a determination of whether maintenance of treatment effects would be achieved. With reading passages, the multipleprobe design is, however, very sensitive to changes that occur as a function of (a) extraneous learning, (b) measurement itself, or (c) generalization of treatment effects across passages. Any one of these reasons may explain the increasing trends in some of the passages. One limitation of the study, however, is that the intervention package was not validated prior to its use by the parents, which increased the risk of an unsuccessful treatment. In addition, due to design constraints comparisons between treatment and control are within passages in the multiple-probe design, leaving open the question about whether experimental control can be established between equal difficulty level but independent control and treatment passages throughout the course of analysis.

\section{EXPERIMENT 2}

\section{Purpose}

In Experiment 2, the experimenter validated the treatment package before training the parent in the use of the package. Moreover, as a part of the experimental analysis the effects of parent implementation were observed directly by the experimenter before the parent used the intervention at home.

\section{Method}

\section{Participants and Settings}

Teachers referred each of the participants based on delayed reading performance. All students were Caucasian and enrolled at an urban Midwestern elementary school. Will was an 8-year and 4-month old, regular education first grade student. Maggie was a 10-year and 2-month old, regular education fourth grade student. Jeremiah was a 10-year and 5-month old, third grade student receiving special education services as a student with a behavior disorder. No participants received remedial or special education services in reading.

The brief experimental analysis occurred in the School Psychologist's office at the elementary school prior to the end of the school year. A round meeting table, approximately 4 feet in diameter was used. The remaining progress monitoring and assessment sessions, which occurred during the summer months, took place throughout various settings in the community (i.e., public library, local restaurant, and the elementary school) in order to accommodate 
the parents' scheduling and transportation constraints. Parents carried out the intervention procedures in their homes.

\section{Materials}

Parents were provided with a micro-cassette recorder and tape, a highlighter, a pencil, copies of the tutoring passages (described below), and a tutoring protocol (available from the third author upon request).

Assessment Passages. Assessment passages were constructed in the same way as those used in Experiment 1. Tutoring assessment passages were those associated with the tutoring condition and had high content overlap with the tutoring passages. They were drawn from the first through fourth grade levels. Control assessment passages were used for the control condition and were of approximately equal difficulty level as the tutoring assessment passages (see screening below), but had low content overlap with the tutoring passages to minimize carryover effects from treatment. In this way, they constituted equivalent but independent stimulus materials. Readability and content overlap information appear in Table 2 .

Tutoring Passages. Tutoring passages were drawn from the same basal reading series as those used in Experiment 1. Passages were also drawn from the first through fourth grade levels. Readability and content overlap information appear in Table 2.

Table 2. Averages (and ranges) for readability, content overlap, and screening results per child in Experiment 2.

\begin{tabular}{|c|c|c|c|c|}
\hline Student & $\begin{array}{l}\text { Stimulus } \\
\text { materials }\end{array}$ & Readability & $\begin{array}{c}\text { Content overlap } \\
\text { with tutoring passage* }\end{array}$ & $\begin{array}{c}\text { Screening } \\
C R W / M\end{array}$ \\
\hline \multicolumn{5}{|l|}{ Jeremiah } \\
\hline & Control Assessment & $4.1(2.3-4.7)$ & $28.8(27.8-39.1)$ & $43.3(26-58)$ \\
\hline & Tutoring Assessment & $4.2(2.7-5.8)$ & $88.6(82.5-91.8)$ & $41.5(30-52)$ \\
\hline & Tutoring & $3.8(2.3-4.9)$ & & \\
\hline \multicolumn{5}{|l|}{ Maggie } \\
\hline & Control Assessment & $5.1(4.3-6.9)$ & $22.8(19.8-38.5)$ & $52.7(45-62)$ \\
\hline & Tutoring Assessment & $5.6(4.3-6.6)$ & $86(80-88.9)$ & $52(43-60)$ \\
\hline & Tutoring & $5.3(4.1-5.8)$ & & \\
\hline Will & Control Assessment & $2.1(1.7-2.9)$ & $20.5(17.9-31.4)$ & $18.3(13-26)$ \\
\hline & Tutoring Assessment & $2(1.5-2.1)$ & $87.5(78.7-98.5)$ & $18.4(7-34)$ \\
\hline & Tutoring & $1.6(1.4-2.1)$ & & \\
\hline
\end{tabular}

*Note: These are reported as percentages. 
Dependent Variable and Interobserver Agreement

As in Experiment 1, CRW/min in the assessment passages served as the dependent variable. Reading assessment sessions were audiotaped in order to evaluate interobserver agreement. A trained graduate student who was blind to the experimental conditions and the assessment results scored $40 \%$ of the weekly assessment sessions (randomly chosen). Interobserver agreement was calculated in the same manner as in Experiment 1. The mean interobserver agreement was $96 \%$ (range, $92 \%$ to $100 \%$ ).

\section{Pre-Experimental Screening}

A pre-experimental screening was conducted to select materials so that they would be of equal difficulty level (according to CRW/min) across conditions (tutoring and control). Participants initially read 26 assessment passages for 1 min each to identify passages that would be included in the study. The passages were then rank ordered according to number of CRW/min. The 14 passages which were closest in difficulty level were assigned semi-randomly in pairs to either the control condition or the tutoring condition. Two control and two tutoring passages were reserved for the brief experimental analysis. The remaining passages were used to evaluate the tutoring intervention (relative to the control condition). Results of the screening for both control and tutoring conditions appear in Table 2.

\section{Instructional and Motivational Variables}

A treatment package containing multiple treatment components was used both in the brief experimental analysis and in the evaluation of the home tutoring intervention. The package included modeling of accurate and fluent reading, repeated practice, error correction, segmenting and blending syllables for difficult words (i.e., words with which repeated errors were made), and access to tangible rewards for meeting performance goals. The entire package was used by the experimenter and parent during the brief experimental analysis. During the tutoring phase of the study the parent was responsible for implementing all of the instructional strategies and the experimenter was responsible for administering the reward contingency based on the assessments conducted by the experimenter. A treatment protocol (available from the third author upon request) was used to prompt correct administration of each step of the intervention.

The sequence of the treatment protocol was as follows. First, the experimenter or parent read the passage to the student (modeling) while the student followed along with his or her finger. Next, the student read the passage 
aloud independently (opportunities to respond). The experimenter or parent highlighted any errors made by the student while the student was reading. Immediately after the student finished reading the passage, the experimenter or parent corrected his or her errors. Specifically, the experimenter or parent showed the marked passage to the student, pointed to each highlighted word and read the word(s) to the student. The student read each word back to the experimenter or parent three times. The experimenter or parent then read the sentence containing the first error word to the student and had the student read the sentence three times aloud. The student read the sentence containing the error word three times.

After receiving modeling, practicing the story once, and having errors corrected, the student then re-read the passage a second time (repeated practice and opportunities to respond) while the parent once again marked errors with a pencil. For words that were incorrectly read yet a second time, the experimenter or parent pointed to each word, sounded out the syllables in the words, and had the student repeat each syllable and blend them to form the correct reading of the word.

The reward contingency was applied to the tutoring assessment passages during each phase of the study. A performance increase of $30 \%$ or greater with three or fewer errors was necessary for the student to earn a tangible reward. The $30 \%$ increase was based on the pre-experimental screening results for that passage (performance increases were individualized by passage). A bracket was placed on the assessment passage immediately after the last word the student had to read to indicate the criterion for reward delivery. During the brief experimental analysis, the experimenter did this immediately after instructional sessions. During the tutoring phase, the experimenter applied the contingency to assessments that were conducted on a weekly basis.

\section{Brief Experimental Analysis and Parent Training}

Experimenter Validation. The first step of the brief experimental analysis was to determine the efficacy of the treatment package through experimenter validation. The experimenter (first author) conducted an instructional trial (using the procedures described above) with the student and then assessed the student's performance in two assessment passages that were of approximately equal difficulty level. One assessment passage had high content overlap with the tutoring passage (tutoring assessment passage) and the other had low content overlap with the tutoring passage (control assessment passage).

Parent Training. The experimenter then trained each parent to implement the reading intervention package. Training was conducted in three steps with both parent and child present. First, the experimenter verbally described the 
components of the intervention and answered any questions from the parent. Second, the experimenter modeled the intervention package with the participant using passages that would not be used during any other portion of the study. Finally, the parent implemented the procedures with his or her child as the experimenter observed. Performance feedback was provided to parents as training was in progress.

Parent Validation. The final part of the brief experimental analysis was to determine whether the parent could achieve the same performance increases as the experimenter. Parents conducted an instructional trial without receiving performance feedback from the experimenter. Once the procedures were complete, the experimenter then assessed student performance in two equal difficulty level assessment passages, a tutoring assessment passage and a control assessment passage.

\section{Experimental Design and Procedures for Parent Tutoring}

An adapted alternating treatments design (Sindelar, Rosenberg, \& Wilson, 1985) was used to evaluate the effectiveness of the treatment package relative to a control or non-instructional condition. With an adapted alternating treatments design, experimental control is established when data series are clearly discriminable from one another. In other words, demonstration of experimental control is contingent on a clear separation of effects across conditions.

Parent Tutoring. Once parent training and intervention validation was complete, the parents were instructed to conduct tutoring sessions four times each week on four separate days. Instruction occurred in one story per week. The experimenter provided a new intervention protocol, tape, and story materials after each weekly assessment.

Weekly Assessments and Reward Contingency. Assessments were conducted separately by the experimenter at the end of the week. In each assessment session, the student read one tutoring assessment passage and one control assessment passage in random order for $1 \mathrm{~min}$ each. The reward contingency was in effect during these assessments.

Intervention Modification. Will's reading fluency level and the number of errors made during baseline (Figure 4) led to an adjustment to the intervention protocol. Instead of reading the entire passage, Will read for $2 \mathrm{~min}$. This change was necessary to prevent negative escape behaviors anticipated by his father who was doing the tutoring. 


\section{Results and Discussion of Experiment 2}

Brief Experimental Analysis

Jeremiah's results are displayed in Figure 3, which represents both the initial screening results for both conditions (white bars) and results after the instructional trial for each passage. Striped bars represent the control condition. Black bars represent the instructional condition. Jeremiah's increase in the treatment
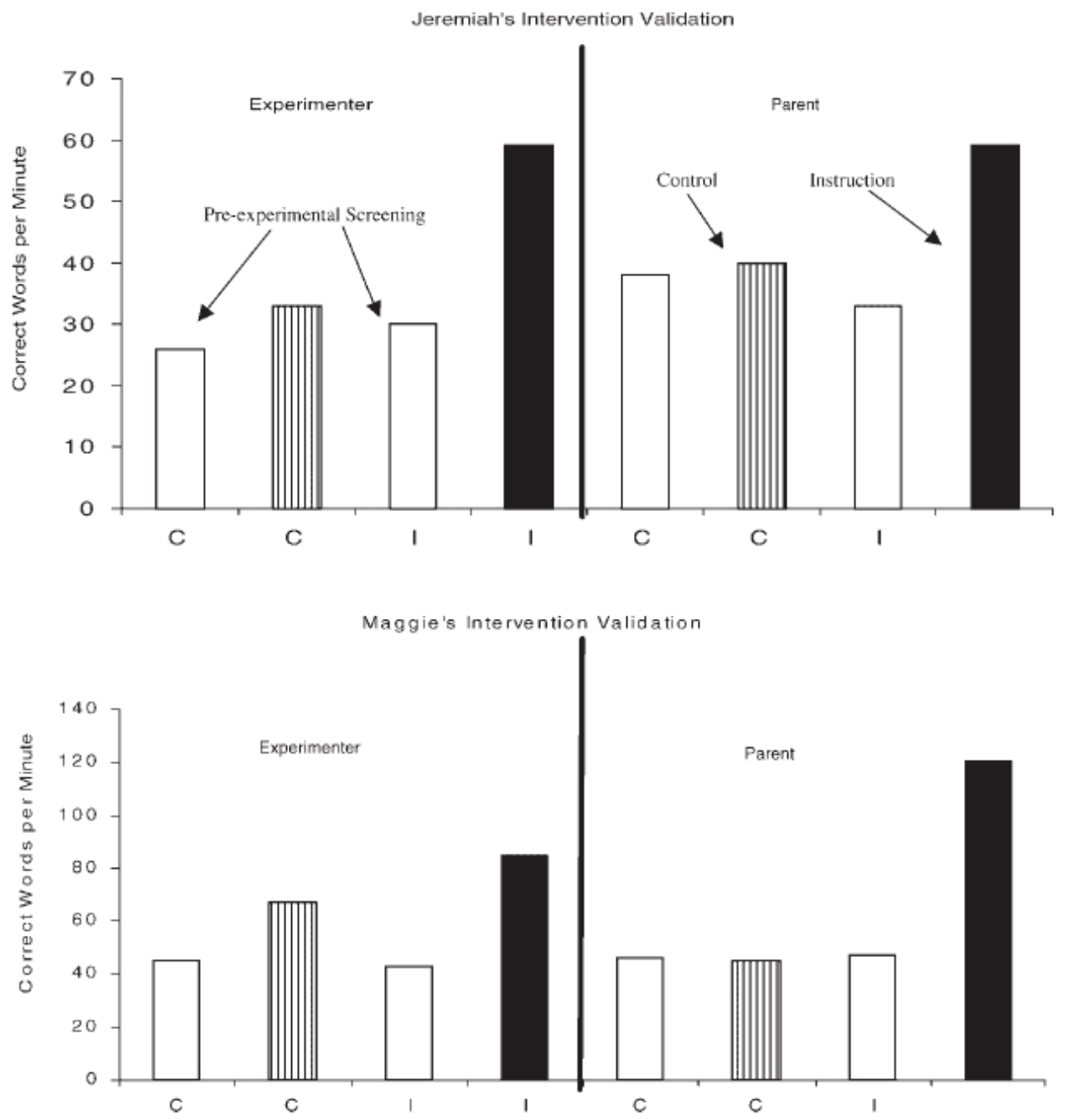

Figure 3. CRW and errors per min during the brief experimental analysis for Jeremiah and Maggie. 
assessment passage (from $30 \mathrm{CRW} / \mathrm{min}$ to $59 \mathrm{CRW} / \mathrm{min}$ ) clearly exceeded the small increase observed in the control assessment passage (26 CRW/min to 33 $\mathrm{CRW} / \mathrm{min}$ ), suggesting that the treatment package was effective at improving Jeremiah's reading fluency deficit in this instance. The same result was found when Jeremiah's parent implemented the intervention. Jeremiah's reading performance increased from $33 \mathrm{CRW} / \mathrm{min}$ to $60 \mathrm{CRW} / \mathrm{min}$ on the tutoring assessment passage while only improving from 38 to $40 \mathrm{CRW} / \mathrm{min}$ on the control assessment passage. Therefore, both the experimenter and parent obtained favorable outcomes with the reading intervention package.

Maggie, whose results are displayed in Figure 3 (bottom panel), also increased her performance in the tutoring assessment passages. Whereas Maggie increased from reading 45 to $67 \mathrm{CRW} / \mathrm{min}$ on the control assessment passage she improved from 43 to $85 \mathrm{CRW} / \mathrm{min}$ on the tutoring assessment passage following the experimenter delivered instructional trial. Following the parent delivered instructional trial Maggie's performance increased from 47 to $123 \mathrm{CRW} / \mathrm{min}$ in the tutoring assessment passage while dropping slightly in the control assessment passage (from 46 to $45 \mathrm{CRW} / \mathrm{min}$ ). As with Jeremiah, the experimenter and the parent both achieved relatively strong increases in generalized reading fluency with the treatment package.

Will's findings, which are displayed in Figure 4, were very similar to the others. Will's performance increased from 7 to $30 \mathrm{CRW} / \mathrm{min}$ in the tutoring assessment passage and decreased from $13 \mathrm{CWPM}$ to $9 \mathrm{CRW} / \mathrm{min}$ in the control assessment passage. When Will's parent conducted an instructional trial with him, he increased from 14 to $24 \mathrm{CRW} / \mathrm{min}$ in the tutoring assessment passage and from 13 to $17 \mathrm{CRW} / \mathrm{min}$ in the control assessment passage.

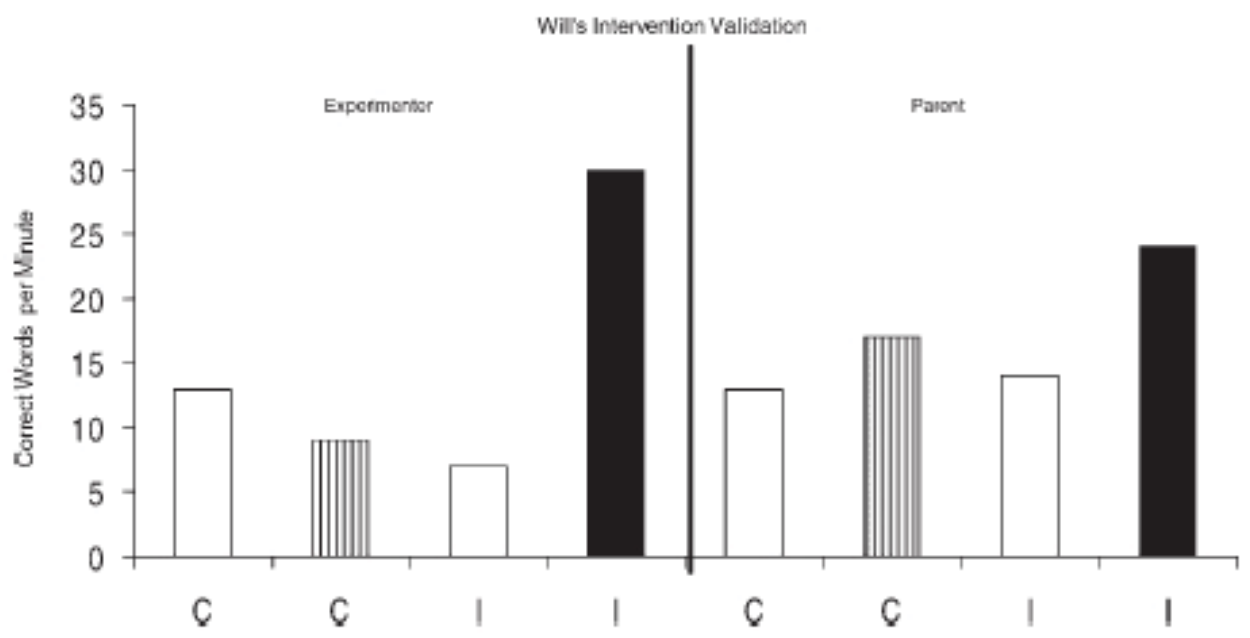

Figure 4. CRW and errors per min during the brief experimental analysis for Will. 
Positive results were obtained for all three participants by both the experimenter and parents. It is interesting to note that all three were reading at somewhat different levels during the initial screening. Maggie, who was the strongest reader initially, showed the highest absolute performance increases. Another interesting finding is that for Maggie and Jeremiah their parents obtained performance increases that matched those of the experimenter. Will's parent obtained findings in the desirable direction; they just were not of the magnitude of those obtained by the experimenter.

\section{Treatment Integrity of Parent Tutoring}

A fifteen-step intervention protocol describing the intervention components was created for parents to follow and to allow for monitoring of implementation (available from the third author upon request). For each treatment component, parents were asked to record step completion. All home sessions were audiotaped by the parents and the tape was returned during the weekly assessment. The tapes were then reviewed by the experimenter and compared to the intervention protocol. A mid-week phone call was made to the parent to provide an opportunity to answer questions and also to update the parents on their performance during the previous week. If there was a negative change in reading performance, the results were presented on a graph to the parent during the following weekly assessment. Parents implemented the reading intervention for 5 weeks. The treatment integrity of parental implementation was $88 \%$ (range, $78 \%$ to $100 \%$ ). In general, treatment integrity was high. Weeks with poor treatment integrity (e.g., missed sessions or missed steps on the protocol) are indicated in the Figures $(5 \& 6)$ as open circles in the tutoring data series.

\section{Parent Tutoring}

All three students demonstrated improvements in reading fluency. Jeremiah's results are displayed in Figure 5. A divergence between the two series indicates that performance on the intervention passages surpassed performance on the control passages across all 5 weeks. However, a decrease in reading performance and a decrease in parental treatment integrity occurred during week 3 . Specifically, Jeremiah's parent failed to implement the intervention a fourth time during the week. Jeremiah read an average of $41 \mathrm{CRW} / \mathrm{min}$ in the control assessment passages across all sessions and an average of $66 \mathrm{CRW} / \mathrm{min}$ in the tutoring assessment passages across all sessions, which represents approximately $50 \%$ more $\mathrm{CRW} / \mathrm{min}$ in the tutoring condition. 


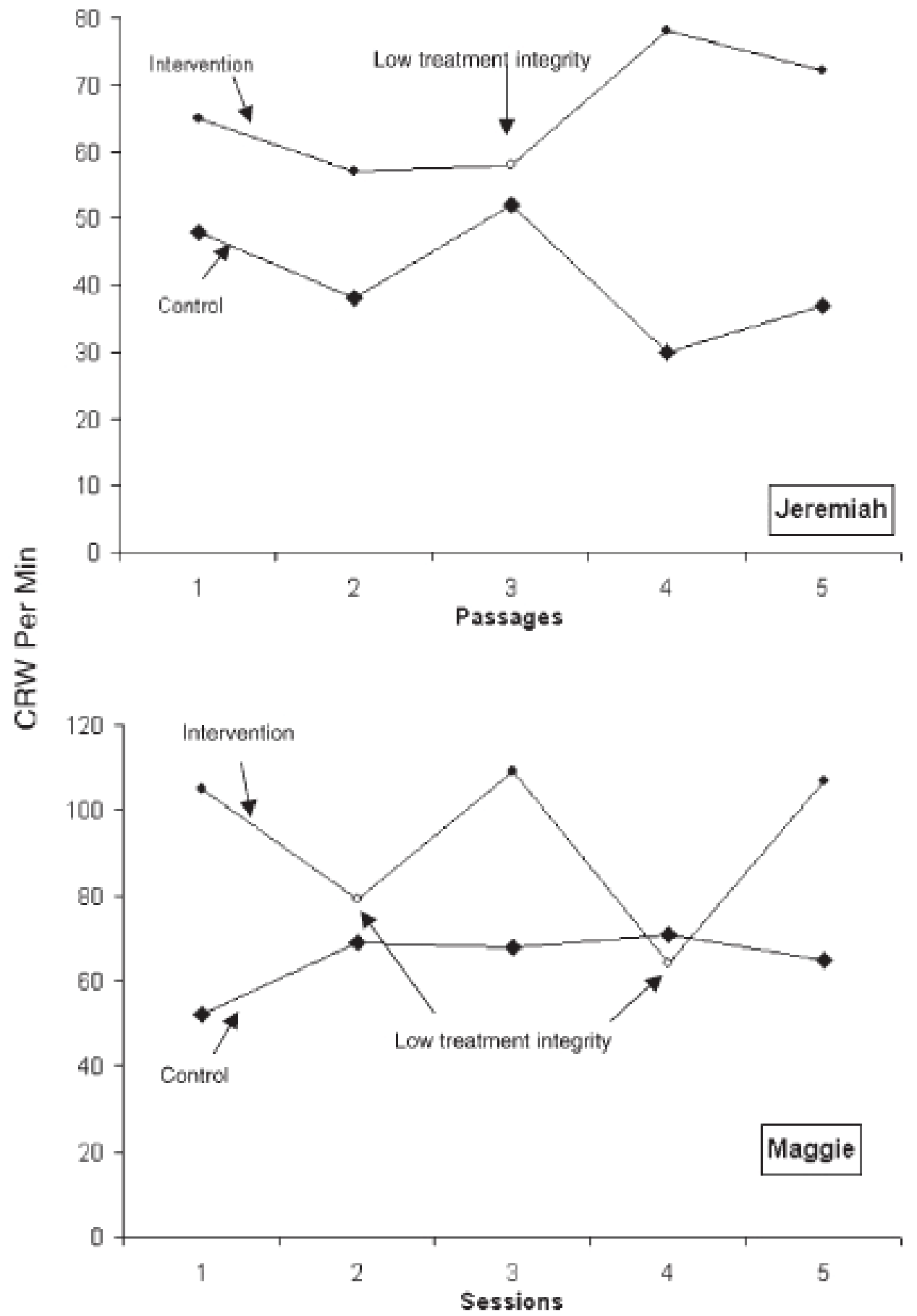

Figure 5. CRW and errors per min during tutoring for Jeremiah and Maggie. 
Maggie's results appear in the bottom panel of Figure 5. Maggie's performance in the tutoring assessment passages surpassed performance on the control passages for four out of the five weeks the intervention was implemented. There was a decrease in treatment integrity and in reading performance during the second and fourth weeks of intervention. During the second week Maggie's parent failed to read the story to her before having Maggie read the story and on the fourth week the intervention was implemented three, not four, times. There was also a two-week time period between the third and fourth intervention passages due to a family vacation. On average, Maggie read $65 \mathrm{CRW} / \mathrm{min}$ in control assessment passages and 92.8 CRW/min in the tutoring assessment passages. Maggie read over $40 \%$ more words on average in the tutoring assessment passages. The result would be even greater if one considered only weeks during which treatment integrity was high.

Will's results are displayed in Figure 6. Data series are clearly discriminable across conditions and there are no overlapping data points. The results indicate that reading fluency in the tutoring assessment passages surpassed reading fluency in the control assessment passages. There was, however, a decrease in reading performance and in treatment integrity during the second and fourth weeks of implementation. During the second week of the intervention, Will's parent failed

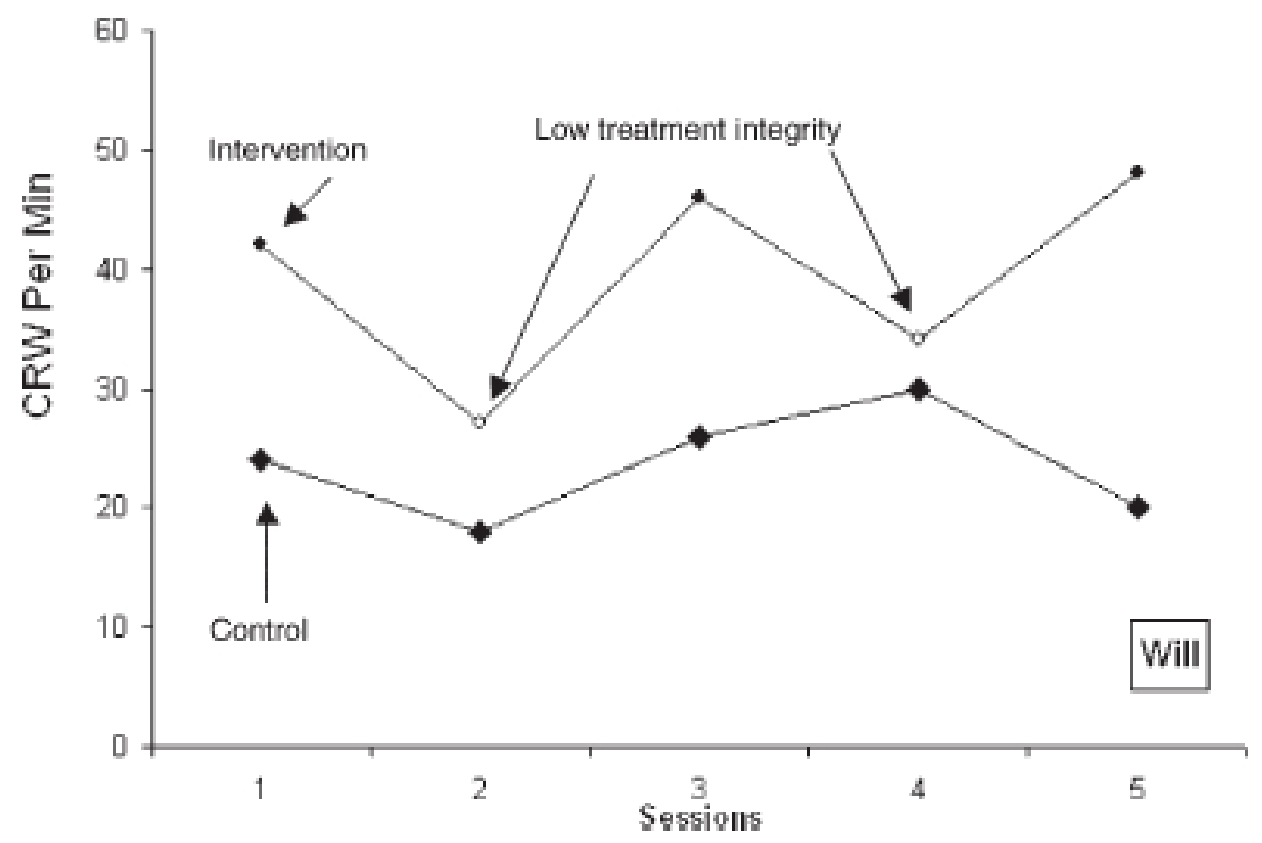

Figure 6. CRW and errors per min during tutoring for Will. 
to implement the error correction component correctly. During the fourth week of the intervention, the tutoring package was only implemented three times. Will read an average of $29.4 \mathrm{CRW} / \mathrm{min}$ in the tutoring passages and $23.6 \mathrm{CRW} / \mathrm{min}$ in the control assessment passages. Will read approximately $25 \%$ more CRW/ min in tutoring passages than in control passages. It is worthy of note that Will demonstrated both the lowest absolute and relative increases in oral reading fluency. Once again, Will's age and reading fluency at the outset were below that of the other participants.

The results across participants clearly support the efficacy of the tutoring intervention package. In addition, tutoring results were strikingly similar to brief experimental analysis results. For example, during the brief experimental analysis Jeremiah read $33 \mathrm{CRW} / \mathrm{min}$ and $40 \mathrm{CRW} / \mathrm{min}$ in the control assessment passages with the experimenter and parent, respectively. During tutoring, Jeremiah read $41 \mathrm{CRW} / \mathrm{min}$ on average in the control assessment passages. On the other hand, Jeremiah read 59 and $60 \mathrm{CRW} / \mathrm{min}$ in the tutoring assessment passages during the brief experimental analysis with the experimenter and parent, respectively. During tutoring, he read $66 \mathrm{CRW} / \mathrm{min}$ on average in the tutoring assessment passages. The very same pattern occurred for the other participants. Admittedly, it was not anticipated that the brief experimental analysis would be predictive of the magnitude as well as the direction of treatment outcomes. Nonetheless, future investigations of brief experimental analyses may find this to be a benefit of the procedures used in this study, especially as refinements are made to the procedures.

\section{GENERAL DISCUSSION}

The results of these studies are consistent with the empirical literature on the potential efficacy of parents as tutors of their children. Across both studies, a combination of instructional and motivational strategies led to increases in oral reading fluency relative to controls both within and across equal difficulty level assessment passages. Positive effects were obtained in spite of slight procedural differences across studies. The results of Experiment 1 suggest that performance increases are likely to be maintained within passages. As noted in the Introduction, parents are in an ideal position to support their child's learning, and behavior analysts have much to offer to maximize the benefits of parent involvement. First, the field of behavior analysis has produced a number of useful heuristics for conceptualizing and identifying effective instruction. Examples include opportunities to respond (Greenwood, Hart, Walker, \& Risley, 1994), active responding (Heward, 1994), and the Instructional Hierarchy (Haring, Lovitt, Eaton, \& Hansen, 1978), each of which has direct implications for the planning and delivery of instruction. These heuristic models allow practitioners to analyze 
specific components of instruction without constraining them to adopt an entire curriculum. This flexibility is especially important when working with parents who want to support their child by working with them for $20 \mathrm{~min}$ in the evening but may not be able to implement a curriculum. Behavior analysts can draw upon simple strategies like modeling, practice, error correction, and performance feedback to supplement the instruction going on in the classroom and thereby increase accuracy, fluency, and generalization of responding.

These heuristic models are also helpful in the training of parents. In both studies, the experimenters generally followed a "tell-show-do-watch-praise" sequence (Martens, 1993), reflecting effective principles of instruction that behavior analysts have used for decades. To further increase the chances of correct implementation, stimulus prompts in the form of tutoring protocols also were used. These protocols described concisely and sequentially the necessary steps for parents. The two studies reported here achieved generally good parent implementation of the procedures. Experiment 2 found a clear and direct correlation between implementation (good or poor) and actual measured outcomes. In this study, the investigator shared graphed data of good and poor outcomes to prompt greater adherence to the tutoring protocol. Future investigations could examine this strategy more systematically.

A very important caveat to this study concerns the effects of the training and support provided by the experimenters. Specifically, it is likely that the effects were achieved in part due to the parents' motivation levels. In all likelihood, the tutoring procedures represented an intrusion into family routines and activities. It is probable that only a very willing parent would repeatedly and consistently follow involved treatment plans like those used in these studies. That said, however, the training and support provided in these two experiments may have capitalized on and reinforced the parents' eagerness to help their children, making them important tools for those wishing to work with parents.

Another hallmark of behavior analysis is repeated measurement over time. This process of response-guided experimentation (Barlow \& Hersen, 1984), a feature of the two studies reported here, may play a significant role in augmenting effects. Support personnel can make adjustments as necessary and, as noted earlier, sharing outcomes with the parent can reinforce performance or signal the need for changes in tutoring routines. Assessment sessions in the current studies also created an opportunity for the experimenters to provide specific performance feedback and reward performance increases. As such, the instructional effects that were obtained cannot be isolated from the motivational variables that were used. Although the failure of these studies to isolate specific treatment effects is a limitation from an analytic perspective, it may more accurately represent the type of partnership that is needed between professionals and parents working together to improve student outcomes. Due to concerns about possible treatment 
implementation problems as these studies were being designed in the first place, we reasoned that if the students were highly motivated to earn a reward, they may even prompt their parents to conduct tutoring sessions. No data were collected on the validity of this assumption. So, these considerations are purely speculative. However, future investigations might look at ways in which student motivation could be used as leverage for improving parent implementation.

From a behavior analytic perspective, the measurement of academic responses like reading words in text provides an index of the degree to which stimulus control is achieved (Daly \& Murdoch, 2000). The use of passages which had high content overlap but which were written as a different story in both investigations was an attempt to measure stimulus control of word reading when stimuli (i.e., words) appear in similar but different conditions (i.e., words appear in different order between tutoring and assessment passages). Students' increases in responding across both studies suggest that they generalized from the training conditions to the assessment conditions. Also, as noted earlier the maintenance data from Experiment 1 suggest that they generalized responding across time within texts as well. Future investigations could examine whether other dimensions of generalization (e.g., eventual increases across time for equal difficulty level texts which have lower content overlap) might be promoted through extended applications of parent interventions.

The other repertoire that behavior analysts bring to the schools is the experimental analysis of behavior. It was especially apparent with Experiment 2 that this repertoire can be readily extended to the development of parent tutoring interventions. The precedent certainly exists in the application of behavior analysis to problem behaviors (Harding, Wacker, Cooper, Millard, \& JensenKovalan, 1994). Indeed, systematically incorporating parent instructional trials into the experimental analysis and treatment validation process may increase the likelihood of choosing effective interventions. One limitation of the procedures used in Experiment 2 was that the treatment package was not dismantled to identify effective treatment components. A component analysis may help the behavior analyst develop a simpler intervention for the parent, which might in turn increase treatment integrity. Another advantage of a component analysis is that it would lead to stronger control conditions as a basis for comparison in follow up analyses. Finally, there may also be other combinations of variables which were not explored in this study which could also produce beneficial outcomes.

Although these studies fail to resolve many questions about the best ways to promote and support parent implemented interventions, this report will have true value if it prompts behavior analysts to use the unique skills they possess in tapping into an excellent resource for increasing students' academic responding in the schools - the children's parents. 


\section{REFERENCES}

Adult Literary Service. (2004). Facts on illiteracy in America. Available on-line: http://indian-river.fl.us/living/services/als/facts.html

Barlow, D. H., \& Hersen, M. (1984). Single case experimental designs: Strategies for studying behavior change (2nd ed.). Boston: Allyn \& Bacon.

Chard, D. J., Vaughn, S., \& Tyler, B. J. (2002). A synthesis of research on effective interventions for building reading fluency with elementary students with learning disabilities. Journal of Learning Disabilities, 35, 386-406.

Christenson, S. L., \& Buerkle, K. (1999). Families as educational partners for children's school success: Suggestions for school psychologists. In C. R. Reynolds, \& T. Gutkin (Eds.), The handbook of school psychology (3rd ed., pp. 709-744). New York: John Wiley \& Sons.

Christenson, S. L., \& Sheridan, S. M. (2001). Schools and families: Creating essential connections for learning. New York: Guilford Press.

Daly, E. J., III, \& Martens, B. K. (1994). A comparison of three interventions for increasing oral reading performance: Application of the instructional hierarchy. Journal of Applied Behavior Analysis, 27, 459-469.

Daly, E. J., III, \& Murdoch, A. (2000). Direct observation in the assessment of academic skill problems. In E. S. Shapiro, \& T. R. Kratochwill (Eds.), Behavioral assessment in schools: Theory, research, and clinical foundations (2nd ed., pp. 46-77). New York, NY: Guilford Publications.

Daly, E. J., III, Lentz, F. E., \& Boyer, J. (1996). The instructional hierarchy: A conceptual model for understanding the effective components of reading interventions. School Psychology Quarterly, 11, 369-386.

Daly, E. J., III, Shroder, J., \& Robinson, A. (2001). Testing treatments for oral reading fluency problems: Two case studies. Proven Practice: Prevention and Remediation Solutions for Schools, 4(1), 2-10.

Daly, E. J., III, Martens, B. K., Kilmer, A., \& Massie, D. (1996). The effects of instructional match and content overlap on generalized reading performance. Journal of Applied Behavior Analysis, 29, 507-518.

Daly, E. J., III, Martens, B. K., Hamler, K. R., Dool, E. J., \& Eckert, T. L. (1999). A brief experimental analysis for identifying instructional components needed to improve oral reading fluency. Journal of Applied Behavior Analysis, 32, 83-94.

Daly, E. J., III, Murdoch, A., Lillenstein, L., Webber, L., \& Lentz, F. E. (2002). An examination of methods for testing treatments: Conducting experimental analyses of the effects of instructional components on oral reading fluency. Education and Treatment of Children, 25, 288-316.

Deno, S. L., \& Mirkin, P. K. (1977). Data-based program modification: A manual. Reston, VA: Council for Exceptional Children.

Duvall, S. F., \& Ward, D. L. (1997). An exploratory study of home school instructional environments and their effects on the basic skills of students with learning disabilities. Education \& Treatment of Children, 20, 150-172.

Duvall, S. F., Delquadri, J. C., Elliott, M., \& Hall, R. V. (1992). Parent-tutoring procedures: Experimental analysis and validation of generalization in oral reading across passages, settings, and time. Journal of Behavioral Education, 2, 281-303.

Eckert, T. L., Ardoin, S. P., Daly, E. J., III, \& Martens, B. K. (2002). Improving oral reading fluency: 
An examination of the efficacy of combining skill-based and performance-based interventions. Journal of Applied Behavior Analysis, 35, 271-281.

Epstein, J. L. (1996). Perspectives and previews on research and policy for school, family, and community partnerships. In A. Booth, \& J. F. Dunn (Eds.), Family-school links-How do they affect educational outcomes? (pp. 209-246). Mahwah, NJ: Lawrence Erlbaum.

Fiala, C. L., \& Sheridan, S. M. (2003). Parent involvement and reading: Using curriculum based measurement to assess the effects of paired reading. Psychology in the Schools, 40, 613-626.

Fuchs, L. S., Fuchs, D., \& Hamlett, C. L. (1989). Effects of instructional use of curriculum-based measurement to enhance instructional programs. Remedial and Special Education, 10, 43-52.

Galloway, J., \& Sheridan, S. M. (1994). Implementing scientific practices through case studies: Examples using home-school interventions and consultation. Journal of School Psychology, 32, 385-413.

Good, R. H., III, Simmons, D. C., \& Smith, S. B. (1998). Effective academic interventions in the United States: Evaluating and enhancing the acquisition of early reading skills. School Psychology Review, 27, 45-56.

Greenwood, C. R., Hart, B., Walker, D., \& Risley, T. (1994). The opportunity to respond and academic performance revisited: A behavioral theory of developmental retardation and its prevention. In R. Gardner III, D. M. Sainato, J. O. Cooper, T. E. Heron, W. L. Heward, J. W. Eshleman, \& T. A. Grossi (Eds.), Behavior analysis in education: Focus on measurably superior instruction (pp. 213-224). Pacific Grove, CA: Brooks/Cole Publishing Co.

Harding, J., Wacker, D. P., Cooper, L. J., Millard, T., \& Jensen-Kovalan, P. (1994). Brief hierarchical assessment of potential treatment components with children in an outpatient clinic. Journal of Applied Behavior Analysis, 27, 291-300.

Haring, N. G., Lovitt, T. C., Eaton, M. D., \& Hansen, C. L. (1978). The fourth R: Research in the classroom. Columbus, OH: Merrill.

Heward, W. L. (1994). Three "low-tech" strategies for increasing the frequency of active student response during group instruction. In R. Gardner III, D. M. Sainato, J. O., Cooper, T. E. Heron, W. L. Heward, J. W. Eshleman, \& T. A. Grossi (Eds.), Behavior analysis in education: Focus on measurably superior instruction (pp. 283-320). Pacific Grove, CA: Brooks/Cole Publishing Co.

Hook, C. L., \& DuPaul, G. J. (1999). Parent tutoring for students with Attention-Deficit/ Hyperactivity Disorder: Effects on reading performance at home and school. School Psychology Review, 28, 60-75.

Juel, D. (1988). Learning to read and write: A longitudinal study of 54 children from first through fourth grades. Journal of Educational Psychology, 80, 437-447.

National Center for Educational Statistics. (2003). National assessment of educational progress. Available online: http://www.nces.ed.gov/naep

National Institute of Child Health and Human Development. (2000). Fluency. In Report of the National Reading Panel. Teaching children to read: An evidence-based assessment of the scientific research literature on reading and its implications for reading instruction: Reports of the subgroups (NIH Publication No. 00-4754). Washington, DC: U.S. Government Printing Office.

National Reading Panel. (2000). Teaching children to read: An evidence-based assessment of the scientific research literature on reading and its implications for reading instruction. Available on-line: http://www.nichd.nih.gov/publications/nrp/smallbook.htm 
Noell, G. H., Freeland, J. T., Witt, J. C., \& Gansle, K. A. (2001). Using brief assessments to identify effective interventions for individual students. Journal of School Psychology, 39, 335-355.

Noell, G. H., Gansle, K. A., Witt, J. D., Whitmarsh, E. L., Freeland, J. T., LaFleur, L. H., Gilbertson, D. N., \& Northup, J. (1998). Effects of contingent reward and instruction on oral reading performance at differing levels of passage difficulty. Journal of Applied Behavior Analysis, 31, 659-664.

O'Shea, L. J., Munson, S. M., \& O'Shea, D. J. (1984). Error correction in oral reading: Evaluating the effectiveness of three procedures. Education and Treatment of Children, 7, 203-214.

Payne, A., Whitehurst, G., \& Angell, A. (1994). The role of home literacy environment in the development of language ability in preschool children from low-income families. Early Childhood Research Quarterly, 9, 427-440.

Pearson, P. D., Johnson, D. D., Clymer, T., Indirsano, R., Venezky, R. L., Baumann, J. F., Hiebert, E., \& Toth, M. (1989). Silver, Burdett, and Ginn. Needham, MA: Silver, Burdett, and Ginn, Inc.

Powell-Smith, K. A., Stoner, G., Shinn, M. R., \& Good, R. H., III. (2000). Parent tutoring in reading using literature and curriculum materials: Impact on student reading achievement. School Psychology Review, 29, 5-27.

Rashotte, C. A., \& Torgesen, J. K. (1985). Repeated reading and reading fluency in learning disabled children. Reading Research Quarterly, 20, 180-188.

Sindelar, P. T., Monda, L. E., \& O’ Shea, L. J. (2001). Effects of repeated readings on instructionaland mastery-level readers. Journal of Educational Research, 83, 220-226.

Sindelar, P. T., Rosenberg, M. S., \& Wilson, R. J. (1985). An adapted alternating treatments design for instructional research. Education and Treatment of Children, 8, 67-76.

Spache, G. (1953). A new readability formula for primary grade materials. Elementary English, 53, 410-413.

Sterling-Turner, H. E., Watson, T. S., Wildmon, M., Watkins, C., \& Little, E. (2001). Investigating the relationship between training type and treatment integrity. School Psychology Quarterly, 16, $56-67$.

U.S. Department of Education's Institute of Education Sciences. (2004). What works clearinghouse. Available online: http://w-w-c.org/about.html

U.S. Department of Education. Office of Educational Research and Improvement. National Center for Education Statistics. The Nation's Report Card: Fourth-Grade Reading 2000, NCES 2001-499 by P. L. Donahue, R. J. Finnegan, A. D. Lutkus, N. L. Allen, and J. R. Campbell. Washington, DC: 2001.

Valleley, R. J., Evans, J. H., \& Allen, K. D. (2002). Parent implementation of an oral reading intervention: A case study. Child and Family Behavior Therapy, 24, 39-50.

Von Brock, M. B., \& Elliott, S. N. (1987). Influence of treatment effectiveness information on the acceptability of classroom interventions. Journal of School Psychology, 25, 131-144.

Weiner, R., Sheridan, S. M., \& Jenson, W. R. (1998). Effects of conjoint behavioral consultation and a structured homework program on math completion and accuracy in junior high students. School Psychology Quarterly, 13, 281-309.

Wilks, R. T., \& Clarke, V. A. (1988). Training versus nontraining of mothers as home reading tutors. Perceptual \& Motor Skills, 67, 135-142.

Wolfendale, S., Topping, K., \& Hewison, J. (1986). A review of parental involvement in children's reading. Educational \& Child Psychology, 3, 55-63. 